\title{
Discussion of Electron Induced Atomic Number Contrast
}

\author{
Lucille A. Giannuzzi
}

\section{L.A. Giannuzzi \& Associates LLC, Fort Myers, FL USA.}

In this paper, atomic number $(Z)$ contrast from electron induced images is revisited. $Z$ contrast obtained with a scanning electron microscope (SEM) is generally associated with the collection of backscattered electrons (BSEs) and is considered to exhibit a monotonic increase in BSE signal with $Z$ [1]. However, there is no monotonic relationship of the backscattering coefficient, $\eta$, with $Z$ at electron energy $\mathrm{E}<5$ $\mathrm{keV}$ [2-5]. This is clearly illustrated in FIG. 1 which plots $\eta$ as a function of $Z$ for $\mathrm{E}<5 \mathrm{keV}$ using data from reference [2].

By convention, all electrons that exit a target at an energy $\leq 50 \mathrm{eV}$ are secondary electrons (SEs) [2]. SEs are usually used to distinguish surface topography since the SE yield increases with increasing specimen tilt angle, $\varphi$, and is proportional to $\sec \varphi$. The low energy SEs cannot travel far and are therefore emitted at an exit depth of only $0.5-1.5 \mathrm{~nm}$ for metals, $10 \mathrm{~nm}$ for carbon, $10-20 \mathrm{~nm}$ for insulators, and up to $30 \mathrm{~nm}$ for metal oxides and alkali halides [2]. It has been shown that the electron induced secondary electron (eSE) signal also varies between different materials $[2,3,5]$ and examples of contrast reversals at low energy are well known [6]. The total SE yield is proportional to the stopping power, diffusion of SEs to the surface and escape of the SEs from the surface [5]. Simplistically, if the stopping power value is large, then the incident electrons will be stopped closer to the target surface generating many SEs yielding a high probability for escaping the target. The stopping power $(\mathrm{eV} / \AA)$ for pure elements, Z, is plotted in FIG. 2 for electron energy $\mathrm{E}<15 \mathrm{keV}[7,8]$. Fig. 2 shows there is a nonmonotonic relationship for electron stopping power across the periodic table for all electron energies presented. The variation in stopping power across the periodic table grows larger as the incident electron energy decreases. The non-monotonic stopping power is indeed proportional to non-monotonic eSE and BSE signals [5]. This non-monotonic behavior is similarly observed for non-monotonic ion induced SE (iSE) emission [5]. Thus there is evidence for non-monotonic $Z$ contrast imaging with both the eSE and BSE signal.

\section{References:}

[1] Scanning Electron Microscopy and X-Ray Microanalysis, eds. Joseph Goldstein et al., $3^{\text {rd }}$ ed., New York, Kluwer (2003).

[2] L. Reimer, Scanning Electron Microscopy: Physics of Image Formation and Microanalysis, $2^{\text {nd }}$ ed., Springer, Berlin, (1998) p. 135-169.

[3] L. Reimer and C. Tollkamp, SCANNING 3, (1980) p.35.

[4] E.H. Darlington and V.E. Coslett, J. Phys. D. 5 (1972) p. 1969.

[5] L.A. Giannuzzi and M. Utlaut, Ultramicroscopy 111 (2011) p.1564.

[6] L. Reimer, Image Formation in Low-Voltage Scanning Electron Microscopy, vol. TT 12, SPIE Optical Engineering Press, Bellingham, Washington, (1993) p. 101.

[7] S. Tanuma C. J. Powell and D. R. Penn, J. of Applied Physics 103, (2008) p. 063707.

[8] S. Tanuma, C. J. Powell and D. R. Penn, Surf. Interface Anal., 37 2005) p. 978. 


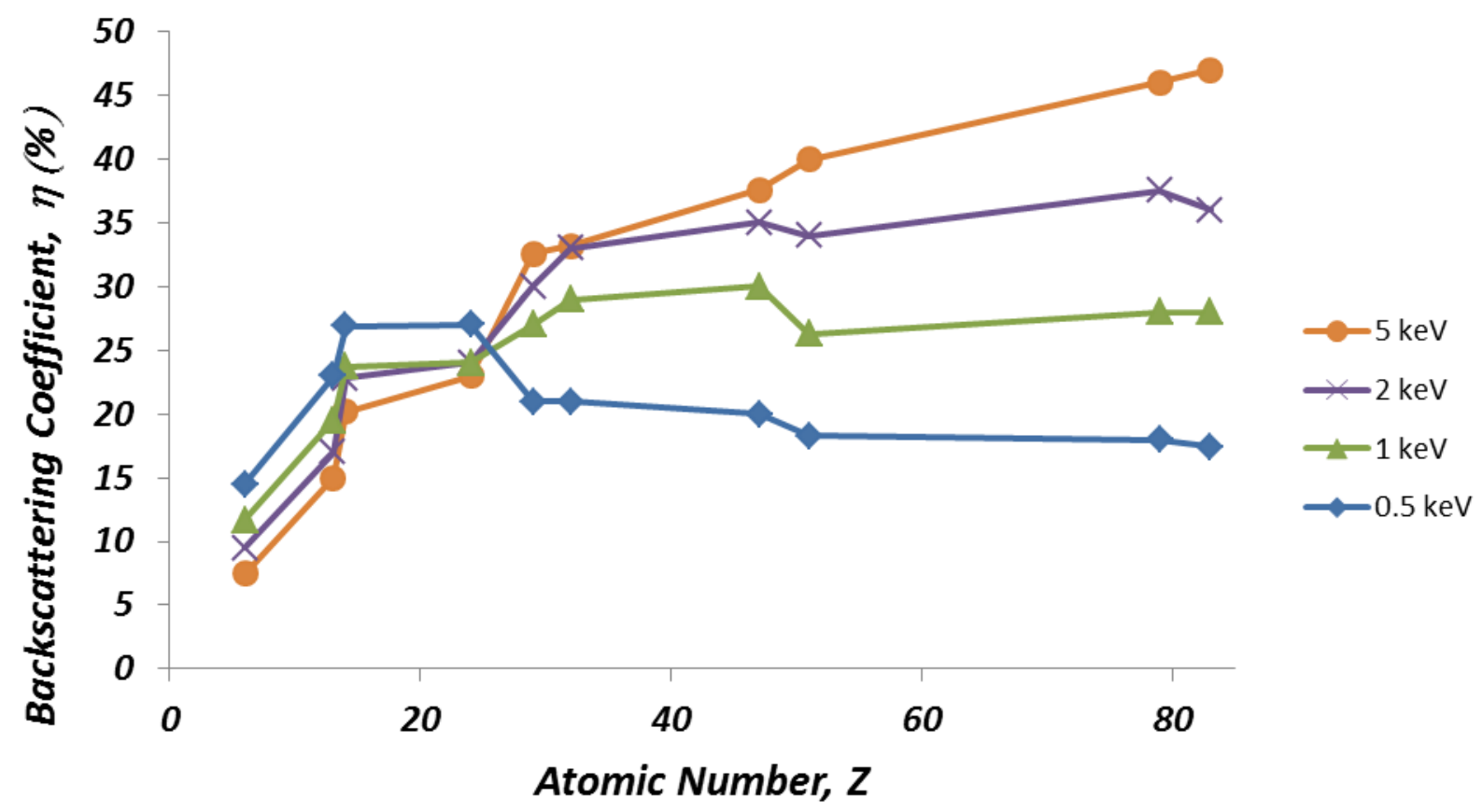

Figure 1. Backscattering coefficient, $\eta$, as a function of atomic number, $Z$, for $\mathrm{E}<5 \mathrm{keV}$ using data from reference [2].

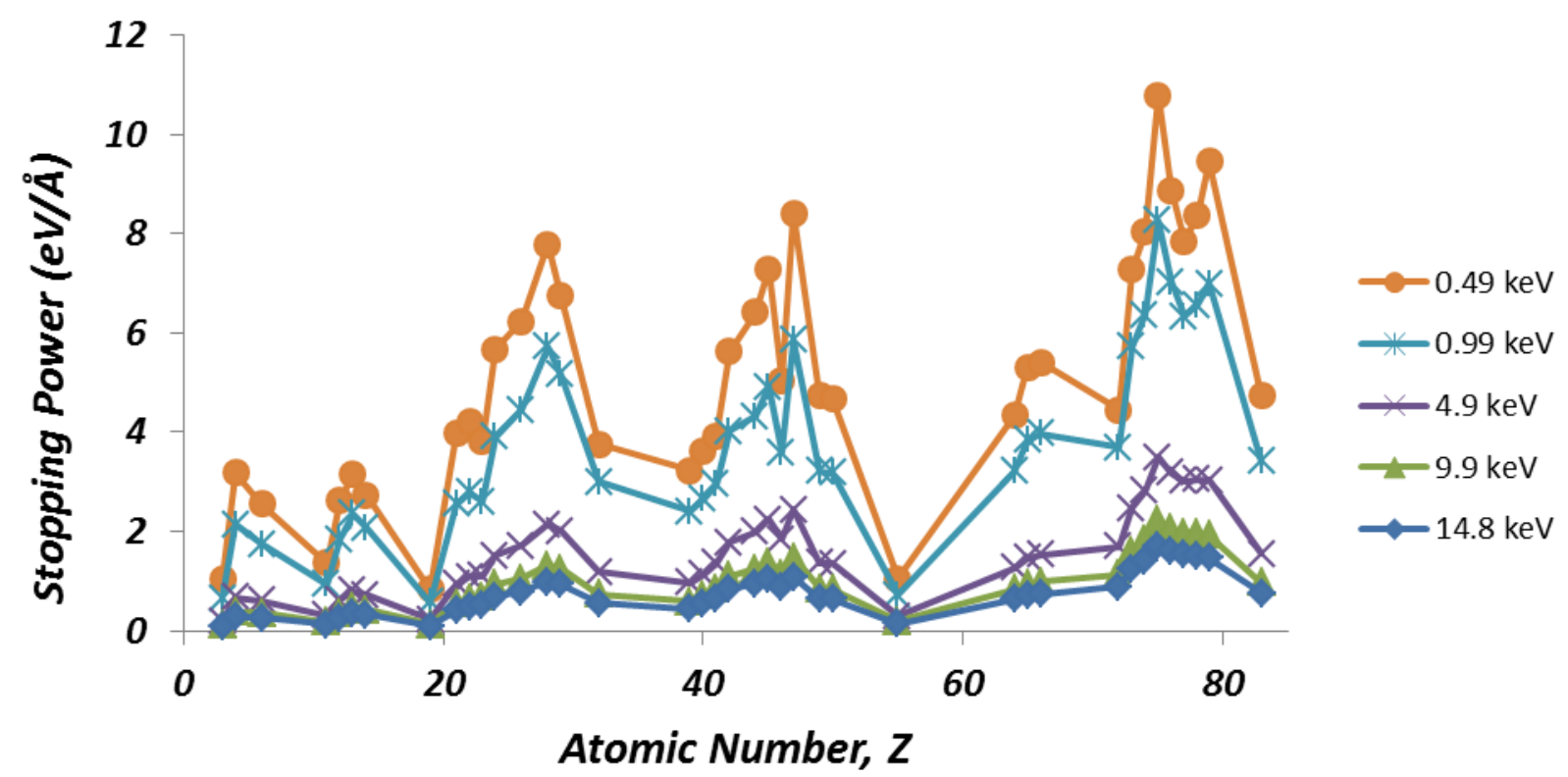

Figure 2. Stopping power $(\mathrm{eV} / \AA)$ as a function of atomic number, $Z$, for electron energy $\mathrm{E}<15 \mathrm{keV}$ using data from references $[7,8]$. 